\title{
INVARIANT MEASURES AND GROWTH CONDITIONS $\left({ }^{1}\right)$
}

\author{
BY
}

\section{JOSE PH MAX ROSENBLATT}

\begin{abstract}
Let $G$ be a finitely-generated group acting on a set $X$ and let $A$ be a nonempty subset of $X$. If $G$ has polynomial growth then there exists a finitelyadditive $G$-invariant positive extended real-valued measure $\mu$ defined on all subsets of $X$ such that $\mu(A)=1$. When $G$ is solvable, it has polynomial growth if and only if it does not contain a free subsemigroup on two generators. If $G$ contains a free subsemigroup $S$ on two generators, then $G$ has exponential growth and there does not exist a measure $\mu$ as above with $G$ acting on itself by multiplication and $A=S$.
\end{abstract}

Introduction. Let $G$ be a group acting on a set $X$. Let $A \subset X$ with $A$ nonempty. The invariant measure problem here is when does there exist a finitelyadditive $G$-invariant measure $\mu$ defined on all subsets of $X$ with values in $[0, \infty]$ such that $\mu(A)=1$ ? When the group $G$ is amenable it is well known that this is equivalent to whenever $a_{1}, \cdots, a_{n} \in R$ and $g_{1}, \cdots, g_{n} \in G$ then $\sum_{i=1}^{n} a_{i} \chi_{g_{i} A} \geq 0$ implies $\sum_{i=1}^{n} a_{i} \geq 0$. We say a group $G$ is supramenable if whenever $G$ acts on a set $X$ and $A \subset X$ is nonempty, there exists a measure $\mu$ as above.

For solvable groups supramenability is nicely related to certain growth conditions in the group. We say the group $G$ is exponentially bounded when for any finite symmetric $F$ $C G$ with $e \in F$ we have $\left(\operatorname{card} F^{N}\right)^{1 / N}$ tends to 1 as $N \rightarrow \propto$. If $G$ is exponentially bounded then it is supramenable. Nilpotent groups have this property and are therefore supramenable. For finitely-generated solvable groups either we have a nilpotent subgroup of finite index and the group is supramenable or there is a subsemigroup on two free generators. These cases are mutually exclusive since, in the first, for all finite symmetric $F \subset G$ with $e \in F$ there exists $K, L>$ 0 such that $\operatorname{card}\left(F^{N}\right) \leq K N^{L}$ for all $N \geq 1$; while, in the second, if $a$ and $b$ generate a free subsemigroup then card $\left(\{a, b\}^{N}\right) \geq 2^{N}$ for all $N \geq 1$. Also, any group $G$ which contains some free subsemigroup $S$ generated by $a$ and $b$ is not supramenable in an obvious way since $a S \cup b S \subset S$ while $a S$ is disjoint from $b S$ and so there does not exist a $G$-invariant finitely-additive measure $\mu$ on $G$ such that $\mu(S)=1$. It follows from this that a solvable group is either

Received by the editors October 26, 1972.

AMS (MOS) subject classifications (1970). Primary 20E15, 20M15; Secondary 15A18, $43 \mathrm{~A} 07$.

(1) This paper is based on part of the author's dissertation at the University of Washington under the supervision of Isaac Namioka. 
exponentially bounded and hence supramenable or it contains a free subsemigroup on two generators but not both. This is also true for connected locally compact groups.

0. Preliminaries. We will let $Z, Q, R$, and $C$ denote the integers, the rational numbers, the real numbers, and the complex numbers respectively. Let $Z^{+}$, $Q^{+}, R^{+}$be the positive elements of $Z, Q$, and $R$ respectively. For $c \in C,|c|$ denotes the absolute value of $c$. We will sometimes extend the real numbers by $\infty$ with $a+\infty=\infty$ for all $a \in R \cup\{\infty\}$. For $p \in Z^{+}, R^{P}=\left\{\left(r_{1}, \ldots, r_{p}\right): r_{1}, \ldots\right.$, $\left.r_{p} \in R\right\}$ is $p$-dimensional Euclidian space.

$C^{P}=\left\{\left(c_{1}, \ldots, c_{p}\right): c_{1}, \cdots, c_{p} \in C\right\}$ and $Z^{p}=\left\{\left(z_{1}, \ldots, z_{p}\right): z_{1}, \cdots, z_{p} \in Z\right\}$.

Given a set $X$ and a subset $A$ of $X$ by $\chi_{A}$ we will denote the characteristic function of $A$ defined on $X$ by $\chi_{A}(x)=1$ if $x \in A, \chi_{A}(x)=0$ if $x \notin A$. $\|A\|$ will denote the cardinality of a finite set $A$. If $S$ is a finite sequence $\left(s_{1}, \ldots, s_{n}\right)$ then $\|s\|=n$. For a set $A \subset X$ and a finite sequence $S=\left(s_{1}, \ldots, s_{n}\right),\|A \cap S\|$ $=\sum_{i=1}^{n} X_{A}\left(s_{i}\right)$. This is consistent with the case that $S$ has no repetitions and is considered as a finite set. For sets $A$ and $B, A \Delta B=(A \backslash B) \cup(B \backslash A)$.

For general group theory see Rotman [18] and Magnus, Karass, and Solitar [12]. We say a semigroup $S$ generated by distinct elements $x_{1}, \ldots, x_{n}$ is freely generated by $x_{1}, \cdots, x_{n}$ if and only if whenever $x_{i_{1}} \cdots x_{i_{k}}=x_{r_{1}} \cdots x_{r_{t}}$ then $k=$ $t$ and $\left(i_{1}, \cdots, i_{k}\right)=\left(r_{1}, \cdots, r_{t}\right)$.

Greenleaf $[5]$ is a good reference for the subject of amenable groups and for von Neumann's work introducing the subject of $G$-invariant finitely-additive meaures. As in Greenleaf [5] a finitely-additive measure on a set $X$ is a function $\mu$ defined on all subsets of $X$ with values in $[0, \infty]$ such that when $A$ and $B$ are disjoint subsets of $X, \mu(A \cup B)=\mu(A)+\mu(B)$. When a group $G$ acts on $X$ then $\mu$ is $G$-invariant if $\mu(g S)=\mu(S)$ for all $S \subset X$ and $g \in G$.

1. The translate property. The problem of the existence of a $G$-invariant finitely-additive measure on $X$ can be stated in terms of functional analysis. Let $G$ be a group acting on a set $X$. Let $A$ be a nonempty subset of $X$. We say a subset $B$ of $X$ is $A$-bounded provided that there exists $g_{1}, \cdots, g_{n} \in G$ with $\bigcup_{i=1}^{n} g_{i} A \supset B$. Given a function $f: X \rightarrow R$ by supp $f$ we denote $\{x \in X: f(x) \neq 0\}$. Let $B_{A}(X)$ denote the linear space under pointwise addition of all bounded functions $f: X \rightarrow R$ with supp $f A$-bounded. $G$ acts as a group of linear transformations of $B_{A}(X)$ by letting $g f(x)=f\left(g^{-1} x\right)$ for all $g \in G, f \in B_{A}(X)$, and $x \in X$. A linear functional $\phi$ on $B_{A}(X)$ is $G$-invariant if $\phi(g f)=\phi(f)$ for all $g \in G$ and $f \epsilon$ $B_{A}(X)$.

We have the usual vector ordering in $B_{A}(X) ; f \geq 0$ if and only if $f(x) \in R^{+}$for all $x$ $\epsilon X$. A linear functional $\phi$ on $B_{A}(X)$ is then positive if $\phi(f) \geq 0$ whenever $f \geq 0$. 
The following two propositions can be found in Greenleaf [5].

1.1 Proposition. There is a G-invariant finitely-additive measure $\mu$ on $X$ such that $\mu(A)=1$ if and only if there is a $G$-invariant positive linear functional $\phi$ on $B_{A}(X)$ sucb that $\phi\left(\chi_{A}\right)=1$.

1.2 Proposition. Let $S_{A}$ be the span $\left\{\chi_{g A}: g \in G\right\}$. If $G$ is amenable there is a G-invariant finitely-additive measure $\mu$ on $X$ sucb that $\mu(A)=1$ if and only if there is a G-invariant positive linear functional $\phi$ on $S_{A}$ such that $\phi\left(\chi_{A}\right)=1$.

1.2 Corollary. There is a $G$-invariant positive linear functional $\phi$ on $S_{A}$ such that $\phi\left(\chi_{A}\right)=1$ if and only if when $a_{1}, \cdots, a_{n} \in R$ and $g_{1}, \cdots, g_{n} \in G$, $\sum_{i=1}^{n} a_{i} \chi_{g_{i} A} \geq 0$ implies $\sum_{i=1}^{n} a_{i} \geq 0$.

Proof. The only if part is immediate since $\phi\left(\sum_{i=1}^{n} a_{i} \chi_{g_{i} A}\right)=\sum_{i=1}^{n} a_{i^{\bullet}}$ The converse follows by defining $\phi: \sum_{i=1}^{n} a_{i} \chi_{g_{i} A} \mapsto \sum_{i=1}^{n} a_{i}$.

We say the translate property holds if the condition of Corollary 1.2 holds. We will say there is an invariant for $(G, X, A)$ if the condition of Proposition 1.1 holds. An invariant for $(G, X, A)$ will be any linear functional $\phi$ as in 1.1. It should be understood that whenever $(G, X, A)$ is written we mean $G$ is a group acting on a set $X$ and $A$ is a nonempty subset of $X$.

2. Free subsemigroups and other examples.

2.1 Examples. The most trivial example is when $G$ is any group and $A \subset X$ is a finite set. Let $n=\|A\|$. Then we can define a $G$-invariant finitely-additive measure $\mu$ by $\mu(S)=n^{-1}\|S\|$ for all $S \subset X$ and $\mu(A)$ will be 1 . So we will be interested in general in only infinite subsets $A \subset X$. Also, if the group $G$ is finite and $A$ is arbitrary then we can define a $G$-invariant finitely-additive measure $\mu$ by taking $x_{0} \in X$ fixed and letting $\mu(S)=\left\|S \cap G x_{0}\right\|$. If we choose $x_{0} \epsilon$ $A$ then $v=\mu /\left\|A \cap G x_{0}\right\|$ will be a $G$-invariant finitely-additive measure on all subsets of $X$ with $v(A)=1$. So in general we will also only be interested in infinite groups.

2.2 Examples. Let $G$ be a locally compact Hausdorff topological group. Let $G$ act on itself by the group multiplication. Let $U \subset G$ be a measurable set with $\infty>m(U)>0$ where $m$ is left-invariant Haar measure. Then $(G, G, U)$ has the translate property since whenever $\sum_{i=1}^{n} a_{i} \chi_{g_{i} U} \geq 0$ then

$$
0 \leq \int\left(\sum_{i=1}^{n} a_{i} \chi_{B_{i} U}\right) d m=\sum_{i=1}^{n} a_{i} m\left(g_{i} U\right)=\sum_{i=1}^{n} a_{i} m(U) .
$$

So $\sum_{i=1}^{n} a_{i} \geq 0$. Hence, if the underlying group structure of $G$ is amenable, then there exists an invariant for $(G, G, U)$. 
However, not all locally compact $T_{2}$ groups are amenable. It was shown by von Neumann [19], see also Greenleaf [ $]$ ], that $S O(3, R)$ is a compact Lie group which contains a subgroup on two free generators. Hence, $S O(3, R)$ as a discrete group is not amenable. More is true; if $U \subset S O(3, R)$ is a nonempty open set then $U$ has the translate property since, with respect to Haar measure $m$ on $S O(3, R), m(U)>0$. But $B_{U}(S O(3, R))=l_{\infty}(S O(3, R))$ by compactness of $S O(3, R)$. Therefore there does not exist an invariant $\theta$ for $(S O(3, R), S O(3, R), U)$ since a constant multiple of it would be a left-invariant mean for $S O(3, R)$ as a discrete group which does not exist.

In general, if $G$ is an amenable group and $B \subset G$ with $G B$-bounded then there exists an invariant for $(G, G, B)$. Also any subgroup $B$ of an amenable $G$ will give an invariant for $(G, G, B)$ since subgroups satisfy the translate property. To see this assume $B$ is a subgroup of $G$ and $a_{1}, \ldots, a_{n} \in R, g_{1}, \ldots, g_{n}$ $\epsilon G$ such that $\sum_{i=1}^{n} a_{i} \chi_{B_{i} B} \geq 0$. We can choose $x_{1}, \ldots, x_{m}$ each in a distinct coset among the cosets $\left\{g_{i} B: i=1, \ldots, n\right\}$. Then $0 \leq \sum_{j=1}^{m} \sum_{i=1}^{n} a_{i} \chi_{g_{i} B}\left(x_{j}\right)=$ $\sum_{i=1}^{n} a_{i}$. Hence, when $G$ is an amenable group there exists an invariant for $(G, G, B)$ when $B$ is a subgroup of $G$. This is only slightly stronger than saying subgroups of amenable groups are amenable.

2.3 Examples. The nonexistence of an invariant for ( $S O(3), S O(3), U)$ where $U$ is a nonempty open set is due to the existence of a subgroup on two free generators. A related example is important here. If we consider any $(G, G, S)$ where $S$ is a free subsemigroup on two generators $a$ and $b$ then $a S U$ $b S \subset S$ and $a S$ is disjoint from $b S$. We write this $a S \cup b S \subset S$. In this case there cannot exist an invariant $\theta$ for $(G, G, S)$.

2.4 Proposition. Let $G$ be a group and $A \subset G$ some nonempty subset. When there exists $a, b \in G$ with $a A \cup b A \subset A$ then either $a$ and $b$ generate $a$ free sub. semigroup or there exists $z$ in the subsemigroup generated by $a$ and $b$ such that $z A \subset a A \cap b A$.

Proof. Suppose $a$ and $b$ do not generate a free subsemigroup. Then there exist two formal positive power words $W(x, y) \neq V(x, y)$ such that $W(a, b)=$ $V(a, b)$. If both words are nonempty then they may have the same first entry on the left; but by cancelling on the left we may assume without loss of generality that this is not the case. Now if one of the words is empry, say $W(x, y)$, then $A$ $=e A=V(a, b) A$. Using $a A \cup b A \subset A$ we get $V(a, b) A \subset v A$ where $v$ is the first entry on the left of $V(a, b)$ which exists since $V(x, y) \neq W(x, y)$ and is consequently nonempty. Hence, let $z=a$ if $v=b$ or vice versa; then $z A \subset a A \cap b A$. If neither word is empty then let $v$ and $w$ be the first entry on the left of $V(a, b)$ and $W(a, b)$ respectively. Let $z=W(a, b)=V(a, b)$. Then using $a A \cup b A \subset A$ we 
we have $z A \subset v A$ and $z A \subset w A$. Since $V(x, y)$ and $W(x, y)$ were originally reduced so that $v \neq w$ we have $z A \subset a A \cap b A$.

2.5 Corollary. G contains a free subsemigroup on two generators if and only if there exists $A \subset G$ nonempty and $a, b \in G$ sucb that $a A \cup b A \subset A$. In this case $a, b$ generate a free subsemigroup.

Proof. Immediate from Proposition 2.4 and Example 2.3 which precedes it.

2.6 Corollary. A group $G$ contains a free subsemigroup on two generators if and only if there exists $A \subset G$ nonempty and $g_{1}, \cdots, g_{n} \in G$ with $n \geq 2$ and $\chi_{A}$ $\sum_{i=1}^{n} \chi_{g_{i} A} \geq 0$.

Proof. Corollary 2.5 in disguise.

We can get a slightly stronger version of Proposition 2.4 as a corollary.

2.7 Corollary. Let $G$ be a group and $A \subset G$ some nonempty subset. Assume there exist $g_{1}, \cdots, g_{n} \in G$ with $\bigcup_{i=1}^{n} g_{i} A \subset A$. Then when the subsemigroup $S$ generated by $g_{1}, \cdots, g_{n}$ does not contain a free subsemigroup on two generators there exists $x \in S$ with $x A \subset \bigcap_{i=1}^{n} g_{i} A$.

Proof. Either $g_{1} A \cup g_{2} A \supset x_{2} A$ with $x_{2}$ in the subsemigroup generated by $g_{1}$ and $g_{2}$ or $g_{1}$ and $g_{2}$ generate a free subsemigroup. This is directly from Proposition 2.4. So there exists $x_{2} \in G$ with $x_{2} A \subset g_{1} A \cap g_{2} A$ and $x_{2}$ in the subsemigroup generated by $g_{1}$ and $g_{2}$. The same argument shows there exists $x_{3} \in G$ with $x_{3} A \subset x_{2} A \cap g_{2} A$ and $x_{3}$ in the subsemigroup generated by $x_{2}$ and $g_{2}$. Therefore $x_{3}$ is in the subsemigroup generated by $g_{1}, g_{2}$, and $g_{3}$. Continue inductively to get $x_{1}, \ldots, x_{n}$ in the semigroup generated by $g_{1}, \cdots, g_{n}$ such that $x_{1}=g_{1}$ and each $i \geq 2 x_{i} A \subset x_{i-1} A \cap g_{i} A$. This gives $x=x_{n}$ such that $x A \subset$ $\bigcap_{i=1}^{n} g_{i} A$ and $x$ is the semigroup generated by $g_{1}, \cdots, g_{n^{*}}$.

Corollary 2.6 lends some support to the conjecture that if a group contains no free subsemigroup on two generators, then each $(G, G, A)$ has the translate property. The difficulty is that more complicated cases than that of 2.6 occur. For instance, if $G$ does contain a subsemigroup $S$ freely generated by $a$ and $b$ then we can let $A=a S$ and let $B=A \cup b A \cup b^{2} A$. Then we have $\chi_{B}+\chi_{b^{2} B} \geq \chi_{b B}$ $+\chi_{a B}+\chi_{b^{4} A B}$ and this does not immediately reduce to the simpler form of $\chi_{C} \geq$ $\sum_{i=1}^{n} X_{g_{i} C}$ for $C$ nonempty except that we know exactly where the free subsemigroup is in this case. For this reason we have been unable to find a combinatorial proof of the conjecture above which works for all groups. The purpose of the next two sections is to describe a wide class of groups for which the conjecture is true besides the trivial example of finite groups. 
3. Exponentially bounded groups. In this section we will be interested in a certain growth condition in groups which always gives the translate property.

3.1 Lemma. Any $(G, X, A)$ bas the translate property if for all finite $F \subset G$ and for all $\epsilon>0$ there exists a finite sequence $S_{\epsilon} \subset X$ sucb that for all $f \in F$

$$
\left\|S_{\epsilon} \cap f A\right\| /\left\|S_{\epsilon} \cap A\right\| \epsilon(1-\epsilon, 1+\epsilon) \text {. }
$$

Proof. Let $\sum_{i=1}^{n} a_{i} X_{B_{i} A} \geq 0$. Let $F=\left\{g_{1}, \cdots, g_{n}\right\}$ and choose $S_{\epsilon}$ as above. Summing over $S_{\epsilon}$ we get that $0 \leq \sum_{x \in S_{\epsilon}} \sum_{i=1}^{n} a_{i} \chi_{g_{i} A}(x)=\sum_{i=1}^{n} a_{i}\left\|S_{\epsilon} \cap g_{i} A\right\|$. Dividing by $\left\|S_{\epsilon} \cap A\right\|$ and taking the limit as $\epsilon \rightarrow 0$ we get $0 \leq \sum_{i=1}^{n} a_{i}$

3.2 Definition. Let $G$ be a group. We say $G$ is exponentially bounded if for all finite, symmetric $F \subset G$ with $e \in F$ as $n \rightarrow \infty,\left\|F^{n}\right\|^{1 / n} \rightarrow 1$ where $F^{n}=$ $\left\{f_{1} \cdots f_{n}: f_{i} \in F\right\}$.

3.3 Theorem. Given $(G, X, A)$ where $G$ is exponentially bounded then $(G, X, A)$ bas the translate property.

Proof. Let $x_{0} \in A$. By Lemma 3.1 we will be done if we show that given a finite symmetric set $F \subset G$ with $e \in F$ and $\epsilon>0$ there exists $N \geq 1$ such that for all $f \in F$ we have that

$$
\left\|f F^{N} x_{0} \cap A\right\| /\left\|F^{N} x_{0} \cap A\right\| \epsilon(1-\epsilon, 1+\epsilon) .
$$

A well-known fact for sequences $\left\{a_{n}\right\}$ of positive numbers is that $\lim$ inf $a_{n+1} / a_{n}$ $\leq \lim \inf a_{n}^{1 / n} \leq \lim \sup a_{n}^{1 / n}$. Let $b_{n}=\left\|F^{n} x_{0} \cap A\right\|$ and $a_{n}=b_{2 n^{\circ}}$ Then $a_{n} \geq 1$ and $a_{n}^{1 / n} \leq\left\|F^{2 n}\right\|^{1 / n} \rightarrow 1$. Hence, $\lim$ inf $a_{n+1} / a_{n}=1$ since each $a_{n+1} / a_{n} \geq 1$. Therefore there exists a sequence $n_{k}$ of positive integers such that $\lim _{k} b_{n_{k+2}} / b_{n_{k}}=1$. Since

$$
1+\frac{\left\|\left(F^{n}{ }^{+2} x_{0} \backslash F^{n} k_{x_{0}}\right) \cap A\right\|}{\left\|F^{n}{ }^{n} x_{0} \cap A\right\|}=\frac{\left\|F^{n}{ }^{n+2} x_{0} \cap A\right\|}{\left\|F^{n} k_{x_{0}} \cap A\right\|}
$$

we have

$$
\lim _{k} \frac{\left\|\left(F^{n} k^{+2} x_{0} \backslash F^{n} k_{x_{0}}\right) \cap A\right\|}{\left\|F^{n} k_{x_{0}} \cap A\right\|}=0 .
$$

Hence for all $f \in F$ since $F$ is symmetric 


$$
\begin{aligned}
& \left|1-\frac{\left\|f F^{n}{ }^{+1} x_{0} \cap A\right\|}{\left\|F^{n}{ }^{n+1} x_{0} \cap A\right\|}\right|=\frac{\left\|F^{n_{k}+1} x_{0} \cap A\right\|-\left\|f F^{n_{k}+1} x_{0} \cap A\right\| !}{\left\|F^{n}{ }^{+1} x_{0} \cap A\right\|} \\
& \leq \frac{\left\|\left(F^{n_{k}+1} x_{0} \Delta f F^{n_{k}+1} x_{0}\right) \cap A\right\|}{\left\|F^{n_{k}+1} x_{0} \cap A\right\|} \\
& =\frac{\left.\left\|\left(F^{n_{k}+1} x_{0} \backslash F^{n_{k}+1} x_{0}\right) \cap A\right\|+\| f F^{n_{k}+1} x_{0} \backslash F^{n_{k}+1} x_{0}\right) \cap A \|}{\left\|F^{n_{k}+1} x_{0} \cap A\right\|} \\
& \leq 2 \frac{\left\|\left(F^{n}{ }^{+2} x_{0} \backslash F^{n} k_{x_{0}}\right) \cap A\right\|}{\left\|F^{n} k_{x_{0}} \cap A\right\|} .
\end{aligned}
$$

Thus, for all $\epsilon>0$ there exists $k$ large enough so that, for all $f \in F$, $\left\|f F^{n_{k+1}} x_{0} \cap A\right\| /\left\|F^{n_{k+1}} x_{0} \cap A\right\| \epsilon(1-\epsilon, 1+\epsilon)$.

3.4 Remark. It is worth noting here that we have proved something a lot stronger. We have shown that when $G$ is exponentially bounded then for any finite symmetric set $F$ with $e \in F$ and any $\epsilon>0$ there is $N \geq 1$ and $K=F^{N} x_{0} \subset X$ such that for all $f \in F$ we have $\|(f K \Delta K) \cap A\| /\|K \cap A\|<\epsilon$. It should be understood that implicitly $\|K \cap A\|>0$. As is shown in [17] this is actually equivalent to the existence of an invariant for $(G, X, A)$.

Also, in the previous proof we used only $\lim \inf \left\|F^{2 n}\right\|^{1 / n}=1$. But there was no loss of generality in assuming $\left\|F^{n}\right\|^{1 / n} \rightarrow 1$ since $\lim _{n}\left\|F^{n}\right\|^{1 / n}$ always exists. This follows from an observation of Milnor [14] that if $\gamma: Z^{+} \rightarrow Z^{+}$is nondecreasing and $\gamma(s+t) \leq \gamma(s) \gamma(t)$ for all $s, t \in Z^{+}$then $\gamma(s)^{1 / s}$ always converges as $s \rightarrow \infty$.

3.5 Corollary. If $G$ is an exponentially bounded group then whenever $G$ acts on a set $X$ and $A \subset X$ is a nonempty set there exists an invariant for $(G, X, A)$.

Proof. Combining Proposition 1.2 and Theorem 3.3 we need only show that an exponentially bounded group is amenable. But given a finite symmetric $F \subset G$ with $e \in F$ we can apply Remark 3.4 to $(G, G, G)$ and get for all $\epsilon>0$ there exists $N \geq 1$ such that, for all $f \in F,\left\|f F^{N} \Delta F^{N}\right\| /\left\|F^{N}\right\|<\epsilon$. This Folner criterion implies $G$ is amenable. 
3.6 Remark. In proving Corollary 3.5 we could have gotten away with proving less. If a group $G$ is given such that there exist invariants for all $(G, G, A)$ then whenever $G$ acts on a set $X$ and $A \subset X$ is nonempty, there exists an invariant for $(G, X, A)$. Let $x_{0} \in A$. Define $\pi: G \rightarrow X$ by $\pi(g)=g x_{0}$. Then $\pi$ is equivariant in the sense that $\pi(g b)=g \pi(b)$ for all $g, b \in G$. For any $S \subset G$ let $\pi^{-1}(S)=\{g \in G: \pi(g) \in S\}$. Then $\pi^{-1}(A)$ is nonempty and there exists a finitelyadditive $G$-invariant measure $\mu$ on $G$ such that $\mu\left(\pi^{-1}(A)\right)=1$. Define $\chi(S)=\mu\left(\pi^{-1}(S)\right)$ for all $S C$ $X$. It is easy to check using the equivariance of $\pi$ that $v$ is a finitely-additive $G$-invariant measure on $X$ such that $v(A)=1$. So we could have considered only triples $(G, G, A)$ to get Corollary 3.5.

4. Growth conditions in solvable groups. In $\$ 2$ we saw if a group $G$ contains a free subsemigroup $S$ on two generators, then there does not exist an invariant for $(G, G, S)$. In $\$ 3$ we saw if $G$ is exponentially bounded, then there exists an invariant for $(G, G, A)$ for any nonempty subset $A$ of $G$. In this section we will show that at least for solvable groups, these are the only two cases. Doing this will depend heavily on the work of Wolf [20] and Milnor [15] on growth conditions in solvable groups.

Assume $G$ is generated by a finite set $F$ with $e \in F$ and $F=F^{-1}$.

4.1 Definition. $G$ bas polynomial growth if there exist $K, L>0$ constants such that for all $N \geq 1$ we have $\left\|F^{N}\right\| \leq K N^{L}$. G has exponential growth if there exist $K>0$. and $\gamma>1$ constants sucb that for all $N \geq 1$ we bave $\left\|F^{n}\right\| \geq K \gamma^{N}$.

These definitions of exponential growth and polynomial growth do not depend on which finite symmetric generating set we use. This was shown in Wolf [20], but is originally an observation due to Milnor [14]. It follows that when $\lim \left\|F^{n}\right\|^{1 / n}$ $=\gamma>1$ there exists $\delta>1$ and $N \geq 1$ such that for all $n \geq N\left\|F^{n}\right\| \geq \delta^{n}$. Using Milnor's observation, we replace the generating set $F$ by $F^{N}$, and we see that $\left\|\left(F^{N}\right)^{m}\right\| \geq\left(\delta^{N}\right)^{m}$ for all $m \geq 1$ which implies $G$ has exponential growth. It is not clear that when $\lim _{n}\left\|F^{n}\right\|^{1 / n}=1$ then $G$ has polynomial growth in general. It is true for solvable groups as we will see later. For other details see Emerson and Greenleaf [3].

4.2 Remark. It follows that if $G$ has polynomial growth, it is exponentially bounded since $\left\|F^{n}\right\|=O\left(n^{L}\right)$ for any finite $F \subset G$ and if $G$ has exponential growth, then it is not exponentially bounded.

A solvable group $G$ is polycyclic if every subgroup $H$ of $G$ is necessarily finitely-generated. Wolf [20] gives other characterizations of polycyclic groups, one of the most important of which is there exists a normal series for $G$ with finitely-generated abelian factors. This means there exist $G_{0} \subset G_{1} \subset \cdots \subset G_{n}$ with $G_{0}=(e), G_{n}=G$ and each $G_{i}$ a normal subgroup of $G_{i+1}$ with $G_{i+1} / G_{i}$ a finitely-generated abelian group for all $i=0, \ldots, n-1$. 
4.3 Remark. Two properties of polycyclic groups which follow from the above characterization will be important later. One is that if $H$ is a normal subgroup of $G$ with $H$ and $G / H$ polycyclic, then $G$ is polycyclic. Another is that we can choose generators $\gamma_{1}, \cdots, \gamma_{p}$ of a polycyclic group $G$ such that every element of $G$ is of the form $\gamma_{1}^{n_{1}} \cdots \gamma_{p}^{n_{p}}$ where $n_{1}, \cdots, n_{p} \in Z$.

4.4 Remark. Wolf [20] shows that a polycyclic group either has a nilpotent subgroup of finite index and has polynomial growth or the group has exponential growth. These cases are mutually exclusive. He actually proves a thearem on the polynomial growth of nilpotent groups which shows how to find the constants $K$ and $L$ in the definition. Another theorem in the same source gives criteria in terms of eigenvalues for deciding if a polycyclic group has a nilpotent subgroup of finite index. Milnor [15] shows that if a finitely-generated group is not polycyclic, then $G$ has exponential growth.

One immediate application of these results is that the class of groups with nilpotent subgroups of finite index is included in the class of exponentially bounded groups. In particular, abelian groups are exponentially bounded. A group $G$ which is locally nilpotent, every finite subset generates a nilpotent subgroup, or one which is locally nilpotent up to taking a finite index subgroup, will also be exponentially bounded since our criterion for being exponentially bounded is a local one. Thus, there is a large class of groups $G$ which give invariants for all (G, $X, A)$.

4.5 Definition. A group $G$ is supramenable if and only if whenever $A \subset G$ with $A$ nonempty there exists an invariant for $(G, G, A)$.

Remark. By Remark 3.6 this is the same as having an invariant for all $(G, X, A)$.

We formalize some previous results in this terminology.

4.6 Theorem. All exponentially bounded groups are supramenable. A group with a free subsemigroup on two generators is not supramenable.

Our conjecture is that a group is supramenable if and only if it is amenable and has no free subsemigroups on two generators. The only if part is clear. By the work of Frey [4] an amenable group has no free subsemigroup on two generators if and only if every subsemigroup is amenable. So our conjecture is that a group is supramenable if and only if it is amenable and every subsemigroup is amenable.

Following Wolf [20] and Milnor [15] we will show that at least for solvable groups this conjecture is true. In fact, we will show that a finitely-generated solvable group either is polycyclic, contains a nilpotent subgroup of finite index and thus 
has polynomial growth, or it contains a free subsemigroup on two generators and thus has exponential growth. These cases are mutually exclusive. The conjecture for solvable groups will follow from this. Jenkins [11] has parallel results for connected separable locally compact topological groups. The above also has application in the problem of symmetry of the group algebra; see Jenkins [10] and Hulanicki [8].

Remark. We will not need it later, but any group $G$ will be supramenable if and only if every finitely-generated subgroup is supramenable. To see this first note that each property implies $G$ is amenable, so by Proposition 1.2 we need only verify the respective translate properties. Given $G$ is supramenable, the translate property for $(H, H, A)$ where $A \subset H$ and $H$ is a finitely-generated subgroup of $G$ follows immediately. Suppose we know the translate property for all such $(H, H, A)$. Given $A \subset G$ which is nonempty we need to check the translate property. Let $g_{1}, \ldots, g_{n} \in G$ and $a_{1}, \ldots, a_{n} \in R$. Suppose $\sum_{i=1}^{n} a_{i} X_{g_{i} A} \geq 0$. Let $H$ be the subgroup generated by $g_{1}, \cdots, g_{n}$ and $g_{0}$ where $g_{0} \in A$. Let $A_{0}=$ $A \cap H$. Then we have $\left.\sum_{i=1}^{n} a_{i} \chi_{B_{i} A}\right|_{H} \geq 0$. Since we know $\left.\chi_{B_{i} A}\right|_{H}=\chi_{g_{i}(A \cap H)}$ $=\chi_{g_{i} A}$, we have $\sum_{i=1}^{n} a_{i} \chi_{g_{i} A_{0}} \geq 0$. Since $\left(H, H, A_{0}\right)$ has the translate property, $\sum_{i=1}^{n} a_{i} \geq 0$. Since the $\sum_{i=1}^{n} a_{i} \chi_{g_{i} A}$ was arbitrary, we have that $(G, G, A)$ has the trans late property for any subset $A \subset G, A$ nonempty.

We first consider finitely-generated nonpolycyclic groups.

4.7 Theorem. Let $G$ be a finitely-generated solvable group with no subsemigroup on two free generators. Then $G$ must be polycyclic.

The converse is not true as we will see when we consider polycyclic groups. We need some lemmas before proving the theorem. The proof via the lemmas given here is identical in form to Milnor [15] replacing "not of exponential growth" with "no free subsemigroup on two generators." The only difference is in the following lemma.

4.8 Lemma. Consider an exact sequence $e \rightarrow A \rightarrow B \rightarrow D \rightarrow e$ where $A$ is abelian and $B$ is finitely-generated. If $B$ bas no subsemigroup on two free generators then, for each $a \in A$ and $b \in B,\left\{b^{k} a b^{-k}: k \in Z\right\}$ spans a finitely-gen. erated subgroup of $A$.

Proof. First we show that if $G$ is a group with no free subsemigroup on two generators, then for all $x, y \in G$ there exists a nontrivial relation of the form $x^{n_{1}} y^{m_{1}} \ldots x^{n_{s}} y^{m_{s}}=x^{k_{1}} y^{l_{1}} \ldots x^{k_{t}} y^{l_{t}}$ with $\sum_{i=1}^{s}\left(n_{i}+m_{i}\right)=\sum_{j=1}^{n}\left(k_{j}+l_{j}\right), k_{1}=0$, $n_{1} \neq 0 \neq l_{1}$, and all $n_{k}, m_{k}, k_{j}, l_{j} \geq 0$. To do this find a nontrivial relation with nonnegative powers of the form $x^{n_{1}} y^{m_{1}} \ldots x^{n_{s}} y^{m_{s}}=x^{k_{1}} y^{l_{1}} \ldots x^{k_{t}} y^{l_{t}}$ with $n_{i}, m_{i}, k_{j}, l_{j}>0$ except possibly $n_{1}, k_{1}, m_{s}, l_{t}$. By cancelling terms on the left 
and then multiplying on the right by $x$ or $y$, we can assume without loss of generality that $n_{1}>0, k_{1}=0$, and $l_{1}>0$. This gives two formal positive power words $W_{1}(u, v) \neq W_{2}(u, v)$ with $W_{1}(x, y)=W_{2}(x, y)$ such that $W_{1}$ begins on the left in $u, W_{2}$ begins on the left in $v$. Let $R_{1}(u, v)=W_{1}(u, v) W_{2}(u, v)$ and $R_{2}(u, v)=W_{2}(u, v) W_{1}(u, v)$. Then $R_{1}(x, y)=R_{2}(x, y)$ and $R_{1} \not R_{2}$ as formal words since one begins in $u$ and the other in $v$. Also the lengths are the same.

Now for $b \in B$ and $a \in A$ since we are assuming $b$ and $b a$ do not generate a free subsemigroup, there exists a nontrivial positive power relation of the form

$$
b^{n} 1(b a)^{m}{ }^{m} b^{n_{2}}(b a)^{m} 2 \ldots b^{n} s(b a)^{m} s=(b a)^{l_{1}} b^{k}(b a)^{l} \cdot \cdots b^{k}(b a)^{l}
$$

where $\sum_{i=1}^{s}\left(n_{i}+m_{i}\right)=\sum_{i=1}^{t}\left(k_{j}+l_{j}\right)$ with $k_{1}=0$. So we have a nontrivial relation of the form $b a^{i_{1}} \ldots b a^{i_{m}^{j=1}}=b a^{j_{1}} \ldots b a^{j_{m}}$ where $i_{r}, j_{r}=0,1$ and $i_{1}=0, j_{1}=1$. Then for convenience let $a_{k}=b^{k} a b^{-k}$. We have $b a^{i_{1}} \cdots b a^{i_{m}}=a_{1}^{i_{1}} a_{2}^{i_{2}} \cdots a_{m}^{i_{m}} b^{m}$. Therefore our relation becomes

$$
a_{1}^{i_{1}} \cdots a_{m}^{i_{m}}=a_{1}^{j_{1}} \cdots a_{m}^{j_{m}} \text { or } a_{1}^{i_{1}-j_{1}} \cdots a_{m}^{i_{m}-j_{m}}=e
$$

where not all $i_{r}-j_{r}=0$. Choosing $m$ as small as possible and conjugating by $b^{-1}$ if necessary, we may assume $i_{1} \neq j_{1}$ and $i_{m} \neq j_{m}$. Note that without loss of generality $m \geq 2$ since $m=1$ implies $a_{1}^{ \pm 1}=e$ implies $a=e$.

It follows that $a_{m}$ is a word in $a_{1}, \ldots, a_{m-1}$. Hence, $a_{m+1}$ is a word in $a_{2}, \cdots, a_{m}$ and therefore in $a_{1}, \cdots, a_{m-1}$ also. By induction one can show that $a_{k}$ for $k>0$ is a word in $a_{1}, \cdots, a_{m-1}$. Similarly, $a_{1}$ is a word in $a_{2}, \cdots, a_{m}$ so $a_{0}$ is a word in $a_{1}, \cdots, a_{m-1}$. Then $a_{-1}$ is a word in $a_{0}, \ldots, a_{m-2}$ and therefore in $a_{1}, \cdots, a_{m-1}$. By induction all $k \leq 0, a_{k}$ is a word in $a_{1}, \ldots$, $a_{m-1}$. Hence, $a_{1}, \ldots, a_{m-1}$. generate the subgroup generated by $\left\{a_{k}: k \in Z\right\}=$ $\left\{b^{k} a b^{-k}: k \in Z\right\}$.

We say a group $D$ has a finite presentation when $D$ is presented with a finite number of generators on which there are a finite number of relations needed to define $D$.

\subsection{Lemma. Let $e \rightarrow A \rightarrow B \stackrel{\pi}{\rightarrow} D \rightarrow$ e be exact, $A$ arbitrary, $B$ finitely-} generated. If $D$ has a finite presentation then there exist $a_{1}, \ldots, a_{m} \in A$ such that every element of $A$ is a product of the conjugates of $\left\{a_{j}\right\}$.

Proof. Choose generators $b_{1}, \ldots, b_{k}$ for $B$ and then $\pi\left(b_{1}\right), \ldots, \pi\left(b_{k}\right)$ generate $D$. Since $D$ has a finite presentation, it has a presentation with these elements as generators subject only to a finite number of relations $r_{1}\left(\pi\left(b_{1}\right), \ldots, \pi\left(b_{k}\right)\right)$ $=\cdots=r_{m}\left(\pi\left(b_{1}\right), \cdots, \pi\left(b_{k}\right)\right)=e$. Define $a_{j}=r_{j}\left(b_{1}, \cdots, b_{k}\right)$. The normal subgroup generated by the set of relations $\left\{r_{i}\left(\pi\left(b_{1}\right), \ldots, \pi\left(b_{k}\right)\right)\right\}$ in the presentation of $D$ gives all relations in $D$. Since this normal subgroup is all products of 
conjugates of $\left\{r_{i}\left(\pi\left(b_{1}\right), \cdots, \pi\left(b_{k}\right)\right)\right\}$, it follows by exactness that every element of $A$ can be expressed as a product of the conjugates of $\left\{a_{j}\right\}$.

4.10 Lemma. Let $e \rightarrow A \rightarrow B \stackrel{\phi}{\rightarrow} D \rightarrow$ e be exact, $A$ abelian, $B$ finitelygenerated, and $D$ polycyclic. If $B$ bas no free subsemigroup on two generators then $B$ must be polycyclic.

Proof. As we remarked 4.3 we can choose $\gamma_{1}, \ldots, y_{p}$ generating $D$ such that every element of $D$ can be expressed as a product $\gamma_{1}^{2} \cdots \gamma_{p}^{i_{p}}$ with $i_{1}, \ldots$, $i_{p} \in Z$. Choose $b_{1}, \cdots, b_{p} \in B$ such that $\phi\left(b_{i}\right)=\gamma_{i}$. By Lemma 4.9 there exists $a_{1}, \cdots, a_{m} \in A$ such that every element of $A$ can be expressed as a product of the conjugates of $\left\{a_{j}\right\}$. Since $A$ is abelian each conjugate of $a_{j}$ can be written as some element $\left(b_{1}^{i_{1}} \cdots b_{p}^{i_{p}}\right)^{-1} a_{j}\left(b_{1}^{i_{1}} \cdots b_{p}^{i_{p}}\right)$. Let $A_{0}$ denote the subgroup of $A$ spanned by $a_{1}, \cdots, a_{m}$. Apply Lemma 4.8 to see there exists a finitely-generated group $A_{1}$ spanned by all conjugates $b_{1}^{i} a_{j} b_{1}^{i}$ where $1 \leq j \leq m, i_{1} \in Z$. Apply the same lemma again to each generator of $A_{1}$ and $b_{2} \in B$ to see that all $b_{2}^{-i 2}\left(b_{1}^{-i 1} a_{j} b_{1}^{i 1}\right) b_{2}^{i 2}$ span a finitely-generated group $A_{2}$. By induction $A_{0} \subset A_{1} \subset$ $A_{2} \subset \ldots \subset A_{p}=A$ gives $A$ finitely-generated. Since $A$ is then polycyclic and since $D$ is polycyclic, $B$ must be polycyclic by Remark 4.3.

Proof of Theorem 4.7. Let the series $G=G^{0} \supset G^{1} \supset \ldots \supset G^{s+1}=(e)$ be the commutator series of a finitely-generated solvable group $G$. Then $e \rightarrow G^{S} \rightarrow G$ $\rightarrow G / G^{S} \rightarrow e$ is exact, $G$ is finitely-generated, and $G^{S}$ is abelian. So by Lemma 4.10, if $G / G^{s}$ is polycyclic and $G$ has no subsemigroup on two free generators, then $G$ is polycyclic. $G / G^{s}$ is a finitely-generated solvable group of one shorter commutator series and $G / G^{S}$ has no subsemigroup on two free generators if $G$ has none. From this an easy induction shows that $G$ is polycyclic.

4.11 Examples. If $G$ is not a polycyclic group but is finitely-generated and solvable then there exists a free subsemigroup on two generators. This proof is unfortunately not constructive and so a few examples are in order. Let $G=$ $\left\langle a, b \mid b a b^{-1}=a^{2}\right\rangle$. That is, $G$ is generated by $a$ and $b$ with the relation $b a b^{-1}$ $=a^{2}$. If we let $N=$ subgroup generated by $\left\{b^{k} a^{l} b^{-k}: k, l \in Z\right\}$ then it is not hard to check $N$ is an abelian normal subgroup of $G$ and $G / N$ is cyclic. So $G$ is solvable. But we can see this without a computation by embedding $G$ in another group. Let $G_{R}$ be the group of additive reals $R$ extended by an element $g$ such that $\tau_{g}$, conjugation by $g$, is the automorphism of $R$ given by multiplying by 2 . This group is given by the presentation $\langle R, g|$ for all $\left.r \in R, \mathrm{grg}^{-1}=2 r\right\rangle$. It can be checked that $g$ has $\propto$ order and no relations are imposed on $R$. Thus, we have $G_{R}$ as described. Note that 0 is the identity of $G_{R}$. We get an isomorphism $i$ of $G$ into $G_{R}$ by letting $i(a)=1, i(b)=g$. It is clear $G_{R}$ is a solvable group; therefore $G$ is a solvable group too. The subgroup $N$ of $G$ corresponds to 
$\left\{k / 2^{l}: k, l \in Z\right\}$ under $i$. This subgroup is not finitely-generated and therefore $G$ is not polycyclic and must contain a free subsemigroup on two generators. We will see later in this section why it is natural to suspect $b$ and $b a$ are free semigroup generators in $G$. For now though let $A=\left\{a^{k} b^{l}: l, k \geq 2\right.$ and $\left.k \equiv 0 \bmod 2\right\}$. Then $b a A \cup b A \subset A$, so $b a$ and $b$ generate a free subsemigroup by Corollary 2.5. The disjointness requires knowing that $a$ and $b$ are infinite order. This can be checked easily but also follows from a theorem of Magnus [12, p. 252].

Another good example of a nonpolycyclic finitely-generated solvable group is the group $H$ generated by $x$ and $\left\{Z_{i}: i \in Z\right\}$ with the relations $x^{k} Z_{i} x^{-k}=Z_{i+k}$, $Z_{i} Z_{k}=Z_{k} Z_{i}$. Actually $G$ is generated by $x$ and $Z_{1}$. Also if $N$ is the subgroup generated by $\left\{Z_{i}: i \in Z\right\}, N$ is a free abelian normal subgroup of $G$ with free abelian generators $\left\{Z_{i}: i \in Z\right\}$ and $G / N$ is cyclic. So $H$ is finitely-generated solvable but not polycyclic. Here $x$ and $Z_{1}$ generate a free subsemigroup as an easy argument shows.

Both groups above are simple examples of solvable and hence amenable groups with subsemigroups on two free generators. Höchster [6] was the first to give an example of this phenomenon with a slightly more complicated finitelygenerated solvable group with two elements which he shows generate a free subsemigroup. The example he gives is not polycyclic.

Now we consider polycyclic groups. We will show after some lemmas,

4.12 Theorem. If $G$ is a polycyclic group then $G$ either bas a nilpotent sub. group of finite index or $G$ contains a free subsemigroup on two generators but not botb.

From this we can get the following:

4.13 Corollary. If $G$ is a polycyclic group then either $G$ bas polynomial growth or $G$ contains a free subsemigroup on two generators but not botb.

Proof. As in Remark 4.4 we use Wolf [20] to prove that when the group contains a nilpotent subgroup of finite index, then it has polynomial growth. If there exists a free subsemigroup on two generators $a, b$ then given any generating set $F$ of $G$ with $e \in F=F^{-1}$ we let $F_{0}=F \cup\left\{a, b, a^{-1}, b^{-1}\right\}$. Then $F_{0}$ is the same kind of generating set and $\left\|F_{0}^{m}\right\| \geq\left\|\{a, b\}^{m}\right\| \geq 2^{m}$. Therefore $G$ has exponential growth. As in Remark 4.2 these cases are mutually exclusive.

4.14 Corollary. If $G$ is a solvable group then either $G$ is supramenable or $G$ contains a free subsemigroup on two generators but not botb.

Proof. It is clear both conditions do not occur at once. If we take a finite subset $F \subset G$ with $e \in F$ and $F=F^{-1}$, then the subgroup $H$ generated by $F$ is 
solvable. If $H$ is not polycyclic then it and $G$ contain a free subsemigroup on two generators by Theorem 4.7. If $H$ is polycyclic, then Corollary 4.13 implies that $H$ contains a free subsemigroup on two generators or $H$ has polynomial growth. As in Remark 4.2, since $F$ was arbitrary, $G$ is exponentially bounded or contains a free subsemigroup on two generators. Theorem 4.6 completes the proof of the corollary.

We see now that everything we claimed depends on Theorem 4.12. As in Corollary 4.13, the two conditions are mutually exclusive. We will need several lemmas before giving the proof of Theorem 4.12.

Let $R[x]$ be all polynomials with real coefficients. If $\Gamma$ is a square matrix and $p(x)=\sum_{i=0}^{n} r_{i} x^{i} \in R[x]$ then $P(\Gamma)=\sum_{i=0}^{n} r_{i} \Gamma^{i}$ where $\Gamma^{0}=I$.

4.15 Lemma. Let $\Gamma$ be an $n \times n$ real matrix. Let $\alpha_{1}, \ldots, \alpha_{n}$ be the canonical basis in $R^{n}$. Let $\phi$ be an eigenvalue of $\Gamma$. Then there exists $K_{1}, \cdots, K_{n}$ $\epsilon C$ not all zero such that whenever $P_{i}, Q_{i} \in R[x], i=1, \ldots, n$, with $\sum_{i=1}^{n} P_{i}(\Gamma) a_{i}$ $=\sum_{i=1}^{n} Q_{i}(\Gamma) a_{i}$ then $\sum_{i=1}^{n} P_{i}(\phi) K_{i}=\sum_{i=1}^{n} Q_{i}(\phi) K_{i}$.

Remark. We will see that each $K_{i}$ can be taken to be a polynomial in $\phi$ with coefficients in the smallest subfield of $R$ containing the coefficients of $\Gamma$.

Proof. Given an eigenvalue $\phi$ for $\Gamma, \phi$ is an eigenvalue of the transposed matrix $\Gamma^{T}$. There exists a nonzero vector $v=\sum_{i=1}^{n} K_{i} a_{i}$ with $K_{i} \in C$ such that $\Gamma^{T} v=\phi v$. We claim these $K_{i}$ are what we want. We need only verify that if $\sum_{i=1}^{n} P_{i}(\Gamma) a_{i}=\sum_{i=1}^{n} r_{i} \alpha_{i}$ where $r_{i} \in R$ and $P_{i}(x) \in R[x]$, then $\sum_{i=1}^{n} P_{i}(\phi) K_{i}=$ $\sum_{i=1}^{n} r_{i} K_{i}$. By linearity of the forms and since $\left\{a_{1}, \ldots, a_{n}\right\}$ is a basis, it is easy to see that we need only verify that when $\Gamma^{m} \alpha_{i}=\sum_{j=1}^{n} r_{j} \alpha_{j}$ then $\phi^{m} K_{i}=$ $\sum_{j=1}^{n}{ }^{r}{ }_{j} K_{j}$ for all $m \geq 1$ and for all $i$. In this case, if we write $\Gamma$ as $\left(\Gamma_{i j}\right)$ where $i$ denotes the row and $j$ the column, then $r_{j}=\Gamma_{j i}^{m}$. For $m=1, \Gamma^{T} v=\phi v$ tells us for any $i$ we have $\phi K_{i}=\sum_{j=1}^{n} \Gamma_{i j}^{T} K_{j}=\sum_{j=1}^{n} \Gamma_{j i} K_{j}$. We may therefore assume inductively that $m \geq 2$ and $\phi^{m-1} K_{s}=\sum_{j=1}^{n} \Gamma_{j s}^{m-1} K_{j}$ for all $s$. We then have for all $i$

$$
\begin{aligned}
\phi^{m} K_{i} & =\phi^{m-1}\left(\phi K_{i}\right)=\phi^{m-1} \sum_{j=1}^{n} \Gamma_{j i} K_{j} \\
& =\sum_{j=1}^{n} \Gamma_{j i}\left(\phi^{m-1} K_{j}\right)=\sum_{j=1}^{n} \Gamma_{j i}\left(\sum_{k=1}^{n} \Gamma_{k j}^{m-1} K_{k}\right) \\
& =\sum_{k=1}^{n}\left(\sum_{j=1}^{n} \Gamma_{k j}^{m-1} \Gamma_{j i}\right) K_{k}=\sum_{k=1}^{n} \Gamma_{k i}^{m} K_{k} \cdot \square
\end{aligned}
$$

4.16 Lemma. Let $\chi \in C,|\chi| \geq 3$. Assume we bave two sets of integers 
$0 \leq a_{1}<a_{2}<\cdots<a_{n}$ and $0 \leq b_{1}<b_{2}<\cdots<b_{m}$. If $\sum_{i=1}^{n} \epsilon_{i} \chi^{a_{i}}=\sum_{j=1}^{m} \delta_{j} \chi^{b_{j}}$ where $\epsilon_{i}$ and $\delta_{j}$ are \pm 1 for all $i$ and $j$ then $n=m$ and $\left(a_{1}, \cdots, a_{n}\right)=\left(b_{1}, \cdots, b_{m}\right)$.

Proof. If $a_{n} \neq b_{m}$, then without loss of generality $a_{n}>b_{m}$. Write $\epsilon_{n} \chi^{a_{n}}=$ $\sum_{j=1}^{m} \delta_{j} \chi^{b_{j}}-\sum_{i=1}^{n-1} \epsilon_{i} \chi^{a_{i}}$.

$$
|\chi|^{a}=\left|\epsilon_{n} \chi^{a_{n}}\right| \leq 2 \sum_{s=0}^{a_{n}-1}|\chi|^{s}=2 \frac{|\chi|^{a_{n}}-1}{|\chi|-1}
$$

therefore $|\chi|^{a_{n}} \leq|\chi|^{a_{n}}-1$ since $|\chi| \geq 3$. This is a contradiction and therefore $a_{n}=b_{m}$. If $\epsilon_{n} \neq \delta_{m}$ then without loss of generality $\epsilon_{n}=1$ and $\delta_{m}=-1$. But then

$$
2 \chi^{a_{n}}=\epsilon_{n} \chi^{a_{n}}-\delta_{m} \chi^{b_{m}}=\sum_{j=1}^{m-1} \delta_{j} \chi^{b_{j}}-\sum_{i=1}^{n-1} \epsilon_{i} \chi^{a_{i}} .
$$

So $2|\chi|^{a_{n}} \leq 2\left(|\chi|^{a_{n}}-1\right) /(|\chi|-1)<|\chi|^{a_{n}}$. Therefore $\epsilon_{n}=\delta_{m}$. Cancelling the highest terms of the power series and continuing inductively, we get $n=m$ and $\left(a_{1}, \ldots, a_{n}\right)=\left(b_{1}, \ldots, b_{m}\right)$.

4.17 Theorem. Consider an exact sequence $e \rightarrow Z^{r} \rightarrow G \rightarrow H \rightarrow e$. For each $g \in G$ let $\tau_{g}(x)=g x g^{-1}$ for all $x \in Z^{r}$. If some $g \in G, r_{g}$ bas an eigenvalue $\phi \in C$ with $|\phi| \neq 1$ then $G$ bas a free subsemigroup on two generators.

Proof. Let $g \in G$ with $\tau_{g}$ having an eigenvalue $\phi$ where $|\phi| \neq 1$. Then $\tau_{g^{-1}}$ will have an eigenvalue $1 / \phi$; therefore without loss of generality we assume $|\phi|>1$. Let $\Gamma$ be the matrix of $\tau_{g}$ with respect to the canonical basis $\alpha_{1}, \ldots$, $a_{r^{*}}$ Choose $K_{1}, \cdots, K_{r}$ for $\phi$ as in Lemma 4.15. At least one $K_{i_{0}} \neq 0$. Let $a=$ $a_{i_{0}}$ and $K=K_{i 0^{\circ}}$. Let $x=g^{n} a$ and $y=g^{n}$. We claim that when $n$ is large enough so that $|\phi|^{n} \geq 3$ then $x$ and $y$ generate a free subsemigroup. To prove this let $A$ be the set of elements of $G$ of the form $\left[P\left(\Gamma^{n}\right) a\right] g^{m}$ where $m \geq 1$ and $P$ is a polynomial with coefficients 0 or 1 and no constant term. Notice we are mixing additive and multiplicative notation in $G$. We claim $x A \cup y A \subset A$. First, given any $\left[P\left(\Gamma^{n}\right) a\right] g^{m}$ in $A$ we compute $x\left[P\left(\Gamma^{n}\right) a\right] g^{m}=\left[\left(\Gamma^{n} P\left(\Gamma^{n}\right)+\Gamma^{n}\right) a\right] g^{n+m}$ and $y\left[P\left(\Gamma^{n}\right) a\right] g^{m}=\left[\Gamma^{n} P\left(\Gamma^{n}\right) a\right] g^{n+m}$. Thus, $x A \cup y A \subset A$. If $x A$ intersects $y A$ then for some $m_{1}, m_{2} \geq 1$ and $P, Q$ polynomials with coefficients 0 or 1 and no constant term, we have $\left[\left(\Gamma^{n} P\left(\Gamma^{n}\right)+\Gamma^{n}\right) a\right] g^{n+m 1}=\left[\Gamma^{n} Q\left(\Gamma^{n}\right) a\right] g^{n+m 2}$. This implies that $n+m_{1}=n+m_{2}$ since otherwise there is some $M \geq 1$ with $g^{M} \in Z^{r}$ and thus $\tau_{g}$ would have all eigenvalues of absolute value 1 . Since $n+m_{1}=n+m_{2}$, we can cancel $g^{n+m} 1$ and get $\left(\Gamma^{n} P\left(\Gamma^{n}\right)+\Gamma^{n}\right) a=\Gamma^{n} Q\left(\Gamma^{n}\right) a$. By Lemma 4.15, we have $\left(\phi^{n} P\left(\phi^{n}\right)+\phi^{n}\right) K=\left(\phi^{n} Q\left(\phi^{n}\right)\right) K$. Cancel $K$ and use Lemma 4.16 to get that the polynomials $z P(z)+z$ and $z Q(z)$ are identical. But since $P$ and $Q$ have no 
constant term, this is nonsense. Thus $x A$ and $y A$ are disjoint. This proves the claim $x A \cup y A \subset A$ and Corollary 2.5 implies $x$ and $y$ generate a free subsemigroup.

Remark. The converse of this theorem is not true without more assumption on $H$. For instance let $G$ be $Z^{p} \oplus H, p \geq 1$, where $H$ contains a free subsemigroup on two generators. Then $G$ contains a free subsemigroup on two generators but each $\tau_{g}=$ identity on $Z^{p}$ for $g \in G$.

4.18 Lemma. Given a group of real matrices under composition which is solvable, there exists a subgroup of finite index whicb can be simultaneously uppertriangulated over the complex numbers.

Proof. See Mal'cev [13].

Proof of Theorem 4.12. Let $G$ be a polycyclic group with $G=G^{0} \supset \ldots$ ว $G^{S+1}=(e)$ the commutator series. Assume that $G$ has no subsemigroup on two free generators. We will show that $G$ has a nilpotent subgroup of finite index. We have the exact sequence $e \rightarrow G^{S} \stackrel{i}{\rightarrow} G \rightarrow G / G^{S} \rightarrow e$. For what we are to show without loss of generality $G^{S}$ is free abelian. To see this let $T$ be the subgroup of torsion elements in $G^{S}$. Since $G$ is polycyclic, $T$ is a finite normal subgroup of $G . e \rightarrow G^{S} / T \stackrel{i}{\rightarrow} G / T \rightarrow G / G^{S} \rightarrow e$ is exact if we take $i^{*}$ the quotient of the map i. $G^{S} / T$ is free abelian and $G / T$ is polycyclic and has no free subsemigroup on two generators. Also the commutator series for $G / T$ is $G / T=G^{0} / T$ ว $\ldots \supset G^{S} / T \supset(e)$. If we show there exists a nilpotent subgroup $N$ of finite index in $G / T$ then we have $e \rightarrow T \rightarrow G \stackrel{\pi}{\rightarrow} G / T \rightarrow e$ is exact and $\pi^{-1}(N)$ is a subgroup of finite index in $G$. Let $H \subset \pi^{-1}(N)$ be the centralizer of $T$ in $\pi^{-1}(N)$; $H=\left\{x \in \pi^{-1}(N)\right.$ : conjugation by $x$ on $T$ is the identity $\}$. Since $T$ is finite, $H$ is finite index in $\pi^{-1}(N)$. Therefore $H$ is finite index in $G . H$ is also nilpotent. To see this, let $Z_{H}=$ Center $(H)$; then $T \subset Z_{H}$. The sequence $e \rightarrow Z_{H} \rightarrow H \rightarrow$ $H / Z_{H} \rightarrow e$ is exact where $H / Z_{H}$ is a quotient of $H / T$. But $H / T \subset \pi^{-1}(N) / T \cong$ $N$. Since $N$ is nilpotent, $H / Z_{H}$ is nilpotent which implies $H$ is nilpotent.

Now we are assuming we have $e \rightarrow G^{S} \rightarrow G \stackrel{P}{\rightarrow} G / G^{S} \rightarrow e$ exact and $G^{S}$ free abelian. $G / G^{S}$ is polycyclic of one shorter commutator series than $G$ and has no free subsemigroup on two generators since $G$ does not. By induction, the case $S=0$ being trivial, we may assume there exists a nilpotent subgroup $M$ of finite index in $G / G^{S}$. Then $p^{-1}(M)$ is a subgroup of finite index in $G$ with $p^{-1}(M) / G^{S} \cong M$ which is nilpotent.

Therefore, without loss of generality we have the following to show: given an exact sequence $e \rightarrow Z^{k} \rightarrow G \rightarrow N \rightarrow e$ where $N$ is nilpotent and $G$ has no free subsemigroup on two generators, then $G$ has a nilpotent subgroup of finite index. Each $\tau_{g}$, conjugation by $g \in G$ on $Z^{k}$, extends to an automorphism of $C^{k}$ 
with respect to the canonical basis $a_{1}, \ldots, a_{k}$ of $Z^{k}$. Theorem 4.17 implies all eigenvalues of $\tau_{g}$ are absolute value 1 . Let $U$ be $\left\{\in G: \tau_{g}\right.$ has all eigenvalues 1). By Lemma 4.18 there exists a subgroup $G_{*}$ of $G$ with $Z^{k} \subset G_{*}$ and $\left[G: G_{*}\right]<$ $\infty$ such that the group $\tau\left(G_{*}\right)$ can be put simultaneously in uppertriangular form over $C$. Let $U_{*}=U \cap G_{*}$. We claim $U_{*}$ is nilpotent and the index of $U_{*}$ in $G=\left[G: U_{*}\right]$ $<\infty$.

First, we show $\left[G: U_{*}\right]<\infty$ by showing $\left[G_{*}: U_{*}\right]<\infty$. To do this write each $\tau_{\boldsymbol{g}}=A_{\boldsymbol{g}} U_{\boldsymbol{g}}$ where $A_{\boldsymbol{g}}$ and $U_{\boldsymbol{g}}$ are polynomials over $Q$ in $\boldsymbol{r}_{\boldsymbol{g}}$ such that

(a) $A_{g} U_{g}=U_{g} A_{g}$,

(b) $A_{g}$ is diagonalizable over $C$,

(c) $U_{g}$ has all eigenvalues 1 .

$A_{g}$ and $U_{g}^{g}$ are also uniquely determined by these properties. When we put $\tau\left(G_{*}\right)$ in uppertriangular form, $A_{g}$ becomes the diagonal and $U_{g}-I$ the part above the diagonal. Thus, $A=\left\{A_{g}: g \in G_{*}\right\}$ is an abelian group of automorphisms of $C^{k}$ and $g \mapsto A_{g}$ is a group homomorphism on $G_{*}$ with kernel $U_{*}$. Hence, $G_{*} / U_{*} \cong$ $A$. Each $A_{g} \in A$ has diagonal elements which are the eigenvalues of $r_{g}$. Since $r_{g}$ is an automorphism of $Z^{k}$ these eigenvalues are algebraic integers of absolute value 1. It follows that each is a root of unity; see Borevich and Shafarevich [2, pp. 104-105 or the remark at the end of the proof]. It follows each $A_{g}$ has finite order. Since $A$ is finitely-generated abelian, $A$ is finite and $\left[G_{*}: U_{*}\right]$ $=$ order of $A<\infty$.

In addition we have the exact sequence $e \rightarrow Z^{k} \rightarrow U_{*} \stackrel{\pi}{\rightarrow} U_{*} / Z^{k} \rightarrow e$. $U_{*} / Z^{k}$ is nilpotent since it is a subgroup of $G / Z^{k}$ which is nilpotent. Each $\tau_{u}$ for $u \in U_{*}$ acts on $C^{k}$ and $r\left(U_{*}\right)$ can be simultaneously uppertriangulated over $C$ with respect to some basis $v_{1}, \ldots, v_{k}$. Since each $\tau_{u}$ for $u \in U_{*}$ has eigenvalues 1 , the matrices when in upper-triangular form have 1 's on the diagonal. Let $C_{r}=C v_{1} \oplus \cdots \oplus C v_{r}$. Let $D_{r}=C_{r} \cap Z^{k}$. Each $D_{r}$ is a normal subgroup of $U^{*}$. Let $a \in D_{r+1}$. Then $a=c v_{r+1}+b$ where $c \in C$ and $b \in C_{r}$. So for $u \in U_{*}, r_{u}(a)=\tau_{u}\left(c v_{r+1}\right)+r_{u}(h)=\left(c v_{r \pm 1}+k\right)+\tau_{u}(h)$ for some $k \in C_{r}$. Hence, each $u$, $\tau_{u}(\alpha) \equiv c v_{r+1} \equiv a \bmod C_{r}$. Since $r_{u}(\alpha)-a \in Z^{k} \cap C_{r}=D_{r}, \tau_{u}(\alpha) \equiv a \bmod D_{r}$. This says, each $x \in D_{r+1} / D_{r}$ is in the center of $U_{*} / D_{r}$. Since $D_{k}=Z^{k}$ and $U_{*} / Z^{k}$ is nilpotent, we can get a central series $e \subset D_{1} \subset \ldots \subset D_{k} \subset C_{1} \subset \ldots \subset$ $C_{r}=U_{*}$ where $C_{i}=\pi^{-1}\left(Z_{i}\right)$ with $Z_{i}$ the $i$ th element of a central series for $U_{*} / Z^{k}=U_{*} / D_{k}$. Hence, $U_{*}$ is nilpotent.

Remark. We can give a simple proof here that the group $A$ in the above is finite. We need only show that when $r$ is an automorphism of $Z^{n}$ with eigenvalues $\lambda_{1}, \ldots, \lambda_{n}$ all absolute value 1 then each $\lambda_{i}$ is a root of unity. In this case we have Trace $A^{k}$ is an integer for all $k \geq 1$. Since Trace $A^{k}$ is $\lambda_{1}^{k}+\cdots$ $+\lambda_{n}^{k}, \lambda_{1}^{k}+\cdots+\lambda_{n}^{k}$ is an integer for all $k \geq 1$. If $S^{1}=\{\lambda \in C:|\lambda|=1\}$ then $S^{1} \times \cdots \times S^{1}$ is compact. So the sequence $\left\{\left(\lambda_{1}^{k}, \ldots, \lambda_{n}^{k}\right): k \in Z^{+}\right\}$must have a 
subsequence $\left\{\left(\lambda_{1}^{k_{i}}, \ldots, \lambda_{n}^{k_{i}}\right)\right\}$ which converges. It follows that $\lambda_{1}^{k_{i+1}-k_{i}}, \ldots$, $\left.\lambda_{\eta}^{k_{i+1}-k_{i}}\right) \rightarrow(1, \ldots, 1)$. Thus, there exists a sequence $\left\{l_{i}\right\} \subset Z^{+} \backslash\{0\}$ such that $\lambda_{j}^{l_{i}} \rightarrow 1$ for all $j$. But since $\lambda_{1}^{l_{i}}+\cdots+\lambda_{n}^{l_{i}}$ is an integer for all $i$, there exists $k=l_{i}$ for $i$ sufficiently large such that $\lambda_{1}^{k}+\cdots+\lambda_{n}^{k}=n$. But then $\operatorname{Re} \lambda_{1}^{k}+\cdots+\operatorname{Re} \lambda_{n}^{k}$ $=\operatorname{Re} n=n$ with $k \geq 1$. Since each $\lambda_{j}$ has absolute value $1, \operatorname{Re} \lambda_{j}^{k} \leq 1$ and $\operatorname{Re} \lambda_{j}^{k}=1$ if and only if $\lambda_{j}^{k}=1$. Hence, we must have for all $j, \operatorname{Re} \lambda_{j}^{k}=1$ and therefore $\lambda_{j}^{k}=1$ where $k \geq 1$. Thus, each $\lambda_{j}$ is a root of unity. We quoted Borevich and Shafarevich in the proof of 4.18 because they prove the stronger result, which is interesting in itself, that any algebraic integer of absolute value 1 is a root of unity.

Examples 4.19. We can see now what was going on in Example 4.11 where we considered a special case of $\left\langle a, b \mid b a b^{-1}=a^{m}\right\rangle$. If $|m| \neq 1$ then $G$ is a nonpolycyclic finitely-generated solvable group. It therefore contains a free subsemigroup on two generators. In fact, $b^{2}$ and $b^{2} a$ generate a free subsemigroup since $b^{2} a b^{-2}=a^{m^{2}}, m^{2}>1$; and so if $A=\left\{a^{i} b^{j}: i, j \geq 1\right.$ and $\left.i \equiv 0 \bmod m^{2}\right\}$, then $b^{2} A \cup b^{2} a A \subset A$. But another way of looking at this which compares with Lemma 4.17 is the following. We embed $G$ in $G_{R}$ where $G_{R}=\langle R, g| \mathrm{grg}^{-1}=$ $m r$ for all $r \in R\rangle$ by mapping $a \mapsto 1, b \mapsto g$. This makes it clear that for $|m| \neq$ 1 , the normal subgroup generated by $a$ in $G$ is not finitely-generated since it corresponds to $\left\{k / m^{l}: k, l \in Z\right\}$ in $G_{R}$ which is not finitely-generated. Thus, $G$ is not polycyclic. Also, $\tau_{g}$ on $R$ has an eigenvalue $m,|m| \neq 1$. So as in Lemma $4.17 g^{n}$ and $g^{n} 1$ generate a free subsemigroup on two generators if $|m|^{n} \geq 3$. Since $|m| \geq 2$, we need only take $n=2$. This says $b^{2}$ and $b^{2} a$ generate a free subsemigroup in $G$. Also, if $|m|=1$ then $G$ and $G_{R}$ have nilpotent subgroups of finite index.

Indeed, whenever we have an exact sequence $e \rightarrow R \rightarrow G \rightarrow H \rightarrow e$ and some $\tau_{g}$ with $g \in G$ has an eigenvalue $\lambda, \lambda>1$, then for $\lambda^{n} \geq 3$ we can argue that $g^{n} 1$ and $g^{n}$ generate a free subsemigroup. The argument is easier than in Lemma 4.17 because we can let $A$ be all elements of the form $\mathrm{kg}^{i}$ where $i \geq 1$ and $k$ is a sum of positive powers of $\lambda^{n}$. Then $g^{n} 1 A \cup g^{n} A \subset A$ with disjointness following by an argument about power series analogous to the one in Lemma 4.17.

Similarly, if we are given $e \rightarrow C^{k} \rightarrow G \rightarrow H \rightarrow e$ exact and $\tau_{g}: C^{k} \rightarrow C^{k}$ with eigenvalue $\lambda$ such that $|\lambda|>1$ then we choose $b \in G$ such that $\tau_{b}$ has eigenvalue $\gamma,|\gamma| \geq 3$, and choose any eigenvector $v \in C^{k}$ for $\tau_{g}$ with respect to $\gamma$. Then an argument as above will show $b v$ and $b$ generate a free subsemigroup. These examples are simpler than Lemma 4.17 because we can choose eigenvectors in $G$ itself.

4.20 Corollary. If $G$ is a connected locally compact group, then $G$ either contains a free subsemigroup on two generators or is supramenable but not both. 
Proof. In [21], Balcerzyk and Mycielski show that $G$ is solvable or it contains a free group on a continuum of generators. So if $G$ contains no free subsemigroup on two generators, then $G$ is solvable and Corollary 4.14 finishes the proof.

Remark. We have seen there is some support for the conjecture that an ame $n$ able group is supramenable if and only if it has no free subsemigroup on two generators in Corollary 4.14 and Corollary 4.20. For general amenable groups this is unresolved. Even for groups $G$ which are periodic, every element of finite order, this is unknown. These groups have no free subsemigroup on one generator, but it is not known if they are even amenable. It is possible to construct periodic groups with exponential growth. Another discouraging aspect of this conjecture concerns some common constructions in the category of groups. Both classes, the supramenable groups and the groups with no subsemigroup on two free generators, are closed under factor groups and subgroups. The only part of this that is perhaps not clear is $G$ supramenable implies $G / H$ supramenable for a normal subgroup $H$. But if $\pi: G \rightarrow G / H$ then $G$ acts on $G / H$ by $g \pi(k)=\pi(g k)$. Since $G$ is supramenable by Remark 3.6 there exists an invariant for $(G, G / H, A)$ for any $A$. By the definition of the $G$ action on $G / H$ this shows $G / H$ is supramenable since $A$ is arbitrary.

We have not been able to show that $G \oplus H$ is supramenable if $G$ and $H$ are supramenable. It is clear if $G$ and $H$ are solvable. But the following proposition is true:

4.21 Proposition. If $G$ and $H$ bave no free subsemigroups on two generators, then $G \oplus H$ bas no free subsemigroup on two generators.

Proof. Let $a, b \in H$. Then as in Lemma 4.8 there exist positive power words $S_{1}$ and $S_{2}$ with $S_{1}(a, b)=S_{2}(a, b)$ and with equal lengths such that $S_{1}$ and $S_{2}$ begin on the left with different entries. So let $(x, y)$ and $(w, z) \in G \oplus H$. For $i=1,2$ let $W_{i}(\eta, \xi)=S_{i}\left(R_{1}(\eta, \xi), R_{2}(\eta, \xi)\right)$ where we choose $R_{i}$ and then $S_{i}$ as follows:

(a) $R_{1}$ and $R_{2}$ are positive power words with $R_{1}(x, w)=R_{2}(x, w)$ but $R_{1}$ begins with $\eta$ and $R_{2}$ begins with $\xi$.

(b) $S_{1}\left(R_{1}(y, z), R_{2}(y, z)\right)=S_{2}\left(R_{1}(y, z), R_{2}(y, z)\right)$ where $S_{1}$ and $S_{2}$ are positive power words with lengths the same number $l$ but $S_{1}$ begins with $R_{1}$ and $S_{2}$ with $R_{2}$.

Then 


$$
\begin{aligned}
W_{1}((x, y), & (w, z))=S_{1}\left(\left(R_{1}(x, w), R_{1}(y, z)\right),\left(R_{2}(x, w), R_{2}(y, z)\right)\right) \\
= & \left(S_{1}\left(R_{1}(x, w), R_{2}(x, w)\right), S_{1}\left(R_{1}(y, z), R_{2}(y, z)\right)\right) \\
= & \left(R_{1}(x, w)^{l}, S_{1}\left(R_{1}(y, z), R_{2}(y, z)\right)\right) \\
= & \left(R_{1}(x, w)^{l}, S_{2}\left(R_{1}(y, z), R_{2}(y, z)\right)\right) \\
= & \left(S_{2}\left(R_{1}(x, w), R_{2}(x, w)\right), S_{2}\left(R_{1}(y, z), R_{2}(y, z)\right)\right)=W_{2}((x, y),(w, z)) .
\end{aligned}
$$

Also, $w_{1}(\eta, \xi) \neq w_{2}(\eta, \xi)$ since one begins with $\eta$ and the other with $\xi$. Since $(x, y)$ and $(w, z)$ were arbitrary, $G \oplus H$ has no free subsemigroup on two generators.

\section{BIBLIOGRAPHY}

1. H. Bass, The degree of polynomial growth of finitely-generated nilpotent groups, Proc. London Math. Soc. 25 (1972), 603-614.

2. Z. I. Borevič and I. R. Šafarevič, Number theory, "Nauka", Moscow, 1964; English transl., Pure and Appl. Math., vol. 20, Academic Press, New York, 1966. MR 30\#1080; $33 \# 4001$.

3. W. R. Emerson and F. P. Greenleaf, Asymptotic behavior of products $C^{p}=C+$ $\ldots+C$ in locally compact abelian groups, Trans. Amer. Math. Soc. 145 (1969), 171-204. MR 40 $\# 2780$.

4. A. H. Frey, Studies in amenable semigroups, Dissertation, University of Washington, Seattle, Wash., 1960.

5. F. P. Greenleaf, Invariant means on topological groups and their applications, Van Nostrand Math. Studies, no. 16, Van Nostrand Reinhold, New York, 1969. MR 40 \#4776.

6. M. Höchster, Semigroups of amenable groups, Proc. Amer. Math. Soc. 21 (1969), 363-364. MR 39\#1575.

7. K. Hoffman and R. Kunze, Linear algebra, Prentice-Hall Math. Series, PrenticeHall, Englewood Cliffs, N. J., 1961. MR 23 \#A3146.

8. A. Hulanicki, On symmetry of group algebras of discrete nilpotent groups, Studia Math. 35 (1970), 207-219. MR 43 \#3814.

9. - On positive functionals on a group algebra multiplicative on a subalgebra, Studia Math. 37 (1971), 163-171.

10. Joe Jenkins, On the spectral radius of elements in a group algebra, Illinois J. Math. 15 (1971), 551-554. MR 44 \#4538.

11. - Growth of connected locally compact groups, J. Functional Analysis 12 (1973).

12. W. Magnus, A. Karrass and D. Solitar, Combinatorial group theory: Presentations of groups in terms of generators and relations, Pure and Appl. Math., vol. 13, Interscience, New York, 1966. MR 34 \#617.

13. A. I. Mal'cev, On certain classes of infinite solvable groups, Mat. Sb. 28 (70) (1951), 567-588; English transl., Amer. Math. Soc. Transl. (2) 2 (1956), 1-21. MR 13, 203; 17, 824 . 
14. J. Milnor, $A$ note on curvature and fundamental group, J. Differential Geometry 2 (1968), 1-7. MR 38 \#636.

15. - Growth of finitely-generated solvable groups, J. Differential Geometry 2 (1968), 447-449. MR 39 \#6212.

16. N. Rickert, Groups with the fixed point property, Dissertation, Yale University, New Haven, Conn., 1965.

17. J. Rosenblatt, $A$ generalization of Folner's condition, Math. Scand. (to appear).

18. J. Rotman, The theory of groups. An introduction, Allyn and Bacon, Boston, Mass., 1965. MR 34 \#4338.

19. J. von Neumann, Zur Allgemeinen Theorie der Masses, Fund. Math. 13 (1929), 73116.

20. J. Wolf, Growth of finitely-generated solvable groups and curvature of Riemannian manifolds, J. Differential Geometry 2 (1968), 421-446. MR 40 \#1939.

21. S. Balcerzyk and J. Mycielski, On the existence of free subgroups in topological groups, Fund. Math. 44 (1957), 303-308.

DEPARTMENT OF MATHEMATICS, UNIVERSITY OF BRITISH COLUMBIA, VANCOUVER 8. CANADA 